Accelerator Division

Alternating Gradient Synchrotron Department BROOKHAVEN NATIONAL LABORATORY

Upton, New York 11973

Accelerator Division

Technical Note

AGS/AD/Tech. Note No. 442

Some Calculations of Dispersion in the Booster

\author{
C.J. Gardner
}

August 30, 1996 


\title{
Some Calculations of Dispersion in the Booster
}

\author{
C. J. Gardner
}

\section{Introduction}

Recent modeling $[1,2]$ of the BTA transport line suggests that one may achieve better transport efficiency if the periodic dispersion and its derivative are made to be zero (or near zero) at the point of extraction in the $\mathrm{F} 6$ straight section of the Booster. In principle this can be accomplished by appropriately adjusting the currents in some subset of the booster quadrupoles, and recent searches $[1,2]$ using the MAD code have yielded some possible solutions. The most promising of these requires adjusting the current in a single quadrupole-the horizontal focusing quadrupole at F2. Following are some algebraic calculations meant to supplement the results obtained with the MAD code. We consider first the case of a single thin quadrupole and calculate its effect on the periodic dispersion. The case of four thin quadrupoles arranged in pairs such as those employed in the gamma-transition-jump scheme of the AGS is then considered. The resulting formulae are applied to the manipulation of the periodic dispersion at F6.

\section{Notation}

We use $\mathbf{T}_{i}$ to denote the unperturbed (i.e. without perturbing quadrupoles) transfer matrix for one turn around a ring starting at some point, $s_{i}$, on the design orbit. The perturbed transfer matrix for one turn starting at $s_{0}$ will be denoted by $\mathbf{T}$. For motion in the horizontal plane, these are three-by-three matricies which we partition as follows:

$$
\mathbf{T}_{i}=\left(\begin{array}{cc}
\mathbf{M}_{i} & \mathbf{d}_{i} \\
\mathbf{0} & 1
\end{array}\right), \quad \mathbf{T}=\left(\begin{array}{ll}
\mathbf{M} & \mathbf{d} \\
\mathbf{0} & 1
\end{array}\right)
$$


where

$$
\begin{gathered}
\mathbf{M}_{i}=\mathbf{I} \cos 2 \pi \nu_{0}+\mathbf{J}_{i} \sin 2 \pi \nu_{0}, \quad \mathbf{M}=\mathbf{I} \cos 2 \pi \nu+\mathbf{J} \sin 2 \pi \nu \\
\mathbf{I}=\left(\begin{array}{ll}
1 & 0 \\
0 & 1
\end{array}\right), \quad \mathbf{J}_{i}=\left(\begin{array}{rr}
\alpha_{i} & \beta_{i} \\
-\gamma_{i} & -\alpha_{i}
\end{array}\right), \quad \mathbf{J}=\left(\begin{array}{rr}
\alpha & \beta \\
-\gamma & -\alpha
\end{array}\right),
\end{gathered}
$$

and

$$
\mathbf{d}_{i}=\left(\begin{array}{c}
d_{i} \\
d_{i}^{\prime}
\end{array}\right), \quad \mathbf{d}=\left(\begin{array}{c}
d \\
d^{\prime}
\end{array}\right), \quad \mathbf{0}=\left(\begin{array}{ll}
0 & 0
\end{array}\right) .
$$

Here $\alpha_{i}, \beta_{i}, \gamma_{i}$ are the unperturbed Courant-Snyder parameters at $s_{i}$, and $\nu_{0}$ is the unperturbed horizontal tune. $\alpha, \beta, \gamma$ are the corresponding perturbed parameters at $s_{0}$ and $\nu$ is the perturbed horizontal tune. The unperturbed and perturbed periodic dispersion vectors at $s_{0}$ are denoted by

$$
\mathbf{D}_{0}=\left(\begin{array}{c}
D_{0} \\
D_{0}^{\prime}
\end{array}\right), \quad \mathbf{D}=\left(\begin{array}{c}
D \\
D^{\prime}
\end{array}\right)
$$

These must satisfy

$$
\mathbf{M}_{0} \mathbf{D}_{0}+\mathbf{d}_{0}=\mathbf{D}_{0}, \quad \mathbf{M D}+\mathbf{d}=\mathbf{D}
$$

and therefore

$$
\mathbf{D}_{0}=\left(\mathbf{I}-\mathbf{M}_{\mathbf{0}}\right)^{-1} \mathbf{d}_{0}, \quad \mathbf{D}=(\mathbf{I}-\mathbf{M})^{-\mathbf{1}} \mathbf{d}
$$

The unperturbed transfer matrix from point $s_{i}$ to point $s_{j}$ is given by

$$
\mathbf{U}_{j i}=\left(\begin{array}{cc}
\mathbf{N}_{j i} & \mathbf{n}_{j i} \\
\mathbf{0} & 1
\end{array}\right), \quad \mathbf{N}_{j i}=\left(\begin{array}{cc}
A_{j i} & B_{j i} \\
E_{j i} & F_{j i}
\end{array}\right)
$$

where

$$
\mathbf{n}_{j i}=\left(\begin{array}{c}
n_{j i} \\
n_{j i}^{\prime}
\end{array}\right)=\mathbf{D}_{j}-\mathbf{N}_{j i} \mathbf{D}_{i}
$$

$\mathbf{D}_{i}$ is the (unperturbed) periodic dispersion vector at $s_{i}$, and

$$
\begin{gathered}
A_{j i}=\sqrt{\beta_{j} / \beta_{i}}\left(\cos \Delta \psi+\alpha_{i} \sin \Delta \psi\right), \quad B_{j i}=\sqrt{\beta_{j} \beta_{i}} \sin \Delta \psi \\
E_{j i}=-\left(\frac{\alpha_{j}-\alpha_{i}}{\sqrt{\beta_{j} \beta_{i}}}\right) \cos \Delta \psi-\left(\frac{1+\alpha_{j} \alpha_{i}}{\sqrt{\beta_{j} \beta_{i}}}\right) \sin \Delta \psi
\end{gathered}
$$




$$
F_{j i}=\sqrt{\beta_{i} / \beta_{j}}\left(\cos \Delta \psi-\alpha_{j} \sin \Delta \psi\right) .
$$

Here $\Delta \psi=\psi_{j}-\psi_{i}$ and $\psi_{i}$ is the unperturbed betatron phase advance from $s_{0}$ to $s_{i}$. For the case $i=j-1$ we define

$$
\mathbf{U}_{j}=\mathbf{U}_{j i}, \quad A_{j}=A_{j i}, \quad B_{j}=B_{j i}, \quad E_{j}=E_{j i}, \quad F_{j}=F_{j i} .
$$

The transfer matrix for a thin quadrupole at $s_{i}$ is given by

$$
\mathbf{R}_{i}=\left(\begin{array}{cc}
\mathbf{Q}_{i} & \mathbf{0} \\
\mathbf{0} & 1
\end{array}\right), \quad \mathbf{Q}_{i}=\mathbf{I}+\mathbf{G}_{i}, \quad \mathbf{G}_{i}=\left(\begin{array}{cc}
\mathbf{0} & 0 \\
G_{i} & 0
\end{array}\right)
$$

where $G_{i}$ is the strength of the quadrupole.

Following Courant and Snyder we also define the symplectic conjugate of a two-by-two matrix $\mathbf{A}$ to be

$$
\overline{\mathbf{A}}=-\mathbf{S A}^{\dagger} \mathbf{S}
$$

where

$$
\mathbf{A}=\left(\begin{array}{ll}
A_{11} & A_{12} \\
A_{21} & A_{22}
\end{array}\right), \quad \mathbf{S}=\left(\begin{array}{rr}
0 & 1 \\
-1 & 0
\end{array}\right)
$$

and a dagger denotes the transpose of the matrix. Thus we have

$$
\overline{\mathbf{A}}=\left(\begin{array}{rr}
A_{22} & -A_{12} \\
-A_{21} & A_{11}
\end{array}\right),
$$

and it follows that

$$
\begin{gathered}
\mathbf{A} \overline{\mathbf{A}}=\overline{\mathbf{A}} \mathbf{A}=\left(A_{11} A_{12}-A_{11} A_{22}\right) \mathbf{I}=\mathbf{I}|\mathbf{A}|, \\
\mathbf{A}+\overline{\mathbf{A}}=\left(A_{11}+A_{22}\right) \mathbf{I}=\mathbf{I} \operatorname{Tr} \mathbf{A} .
\end{gathered}
$$

If $|\mathbf{A}|=1$ then (18) implies $\overline{\mathbf{A}}=\mathbf{A}^{-1}$.

\section{Single Quadrupole Perturbation}

Let us now consider a single thin quadrupole placed at a point, $s_{1}$, on the design orbit such that

$$
s_{0}<s_{1}<s_{2}, \quad s_{2}=s_{0}+C
$$


where $C$ is the ring circumference. We wish to calculate the effect of this quadrupole on the periodic dispersion at $s_{0}$. In terms of the notation introduced in the previous section we have

$$
\mathbf{T}_{0}=\mathbf{U}_{2} \mathbf{U}_{1}, \quad \mathbf{T}_{1}=\mathbf{U}_{1} \mathbf{U}_{2}, \quad \mathbf{T}=\mathbf{U}_{2} \mathbf{R}_{1} \mathbf{U}_{1}
$$

and therefore

$$
\begin{gathered}
\mathbf{M}_{0}=\mathbf{N}_{2} \mathbf{N}_{1}, \quad \mathbf{M}_{1}=\mathbf{N}_{1} \mathbf{N}_{2}, \quad \mathbf{M}=\mathbf{N}_{2} \mathbf{Q}_{1} \mathbf{N}_{1}, \\
\mathbf{d}_{\mathbf{0}}=\mathbf{N}_{2} \mathbf{n}_{1}+\mathbf{n}_{2}, \quad \mathbf{d}_{1}=\mathbf{N}_{1} \mathbf{n}_{2}+\mathbf{n}_{1}, \quad \mathbf{d}=\mathbf{N}_{\mathbf{2}} \mathbf{Q}_{1} \mathbf{n}_{1}+\mathbf{n}_{2},
\end{gathered}
$$

where

$$
\mathbf{n}_{1}=\mathbf{D}_{1}-\mathbf{N}_{1} \mathbf{D}_{0}, \quad \mathbf{n}_{2}=\mathbf{D}_{2}-\mathbf{N}_{2} \mathbf{D}_{1}=\mathbf{D}_{0}-\mathbf{N}_{2} \mathbf{D}_{1} .
$$

(Note that since $s_{2}=s_{0}+C$ we have $\mathbf{D}_{2}=\mathbf{D}_{0}$.) Using (24) in the last of equations (23) we then have

$$
\mathbf{d}=\mathbf{N}_{2} \mathbf{Q}_{1}\left(\mathbf{D}_{1}-\mathbf{N}_{1} \mathbf{D}_{0}\right)+\mathbf{D}_{0}-\mathbf{N}_{2} \mathbf{D}_{1}
$$

and using $\mathbf{Q}_{1}=\mathbf{I}+\mathbf{G}_{1}$ we have

$$
\mathbf{d}=\mathbf{N}_{2} \mathbf{G}_{1} \mathbf{D}_{1}+(\mathbf{I}-\mathbf{M}) \mathbf{D}_{0}
$$

where

$$
\mathbf{M}=\mathbf{N}_{2} \mathbf{Q}_{1} \mathbf{N}_{1}=\mathbf{M}_{0}+\mathbf{N}_{2} \mathbf{G}_{1} \mathbf{N}_{1} \text {. }
$$

The perturbed periodic dispersion vector at $s_{0}$ is then

$$
\mathbf{D}=(\mathbf{I}-\mathbf{M})^{-1} \mathbf{d}=(\mathbf{I}-\mathbf{M})^{-1} \mathbf{N}_{2} \mathbf{G}_{1} \mathbf{D}_{1}+\mathbf{D}_{0}
$$

and therefore

$$
\mathbf{D}-\mathbf{D}_{0}=(\mathbf{I}-\mathbf{M})^{-1} \mathbf{N}_{2} \mathbf{G}_{1} \mathbf{D}_{1} .
$$

Thus (29) and (27) give the difference between the perturbed and unperturbed periodic dispersion vectors at $s_{0}$ in terms of the strength of the thin quadrupole at $s_{1}$ and the unperturbed lattice parameters.

Equation (29) has the same form as the expression for the Periodic Closed Orbit Distortion at $s_{0}$ due to a dipole perturbation at $s_{1}$. With a little more algebra it can be simplified a bit further. Using (2), (18) and (19) we have

$$
(\mathbf{I}-\mathbf{M})(\mathbf{I}-\overline{\mathbf{M}})=2 \mathbf{I}-(\mathbf{M}+\overline{\mathbf{M}})=W \mathbf{I}
$$


where

$$
W=2-\operatorname{Tr} M=2(1-\cos 2 \pi \nu) .
$$

Thus

$$
(\mathbf{I}-\mathbf{M})^{-1}=\frac{1}{W}(\mathbf{I}-\overline{\mathbf{M}})
$$

where

$$
\overline{\mathbf{M}}=\overline{\mathbf{M}}_{0}-\overline{\mathbf{N}}_{1} \mathbf{G}_{1} \overline{\mathbf{N}}_{2} \text {. }
$$

Now, multiplying (33) by $\mathbf{N}_{2} \mathbf{G}_{1}$ and using $\overline{\mathbf{N}}_{2} \mathbf{N}_{2}=\mathbf{I}$ and $\mathbf{G}_{1}^{2}=\mathbf{0}$, we have

$$
\overline{\mathbf{M}} \mathbf{N}_{2} \mathbf{G}_{1}=\overline{\mathbf{M}}_{0} \mathbf{N}_{2} \mathbf{G}_{1}-\overline{\mathbf{N}}_{1} \mathbf{G}_{1}^{2}=\overline{\mathbf{M}}_{0} \mathbf{N}_{2} \mathbf{G}_{1}
$$

and therefore

$$
(\mathbf{I}-\mathbf{M})^{-1} \mathbf{N}_{2} \mathbf{G}_{1}=\frac{1}{W}(\mathbf{I}-\overline{\mathbf{M}}) \mathbf{N}_{2} \mathbf{G}_{1}=\frac{1}{W}\left(\mathbf{I}-\overline{\mathbf{M}}_{0}\right) \mathbf{N}_{2} \mathbf{G}_{1} .
$$

Thus (29) becomes

$$
\mathbf{D}-\mathbf{D}_{0}=\frac{1}{W}\left(\mathbf{I}-\overline{\mathbf{M}}_{0}\right) \mathbf{N}_{2} \mathbf{G}_{1} \mathbf{D}_{1}
$$

and since

$$
\overline{\mathbf{M}}_{0} \mathbf{N}_{2}=\mathbf{N}_{2} \overline{\mathbf{M}}_{1} \text {, }
$$

which follows from (22), we also have

$$
\mathbf{D}-\mathbf{D}_{0}=\frac{1}{W} \mathbf{N}_{2}\left(\mathbf{I}-\overline{\mathbf{M}}_{1}\right) \mathbf{G}_{1} \mathbf{D}_{1} .
$$

To obtain $W$ in terms of the unperturbed parameters we take the trace of (27). Thus

$$
\operatorname{Tr} \mathbf{M}=\operatorname{Tr} \mathbf{M}_{0}+\operatorname{Tr}\left(\mathbf{N}_{2} \mathbf{G}_{1} \mathbf{N}_{1}\right)
$$

where

$$
\begin{aligned}
& \operatorname{Tr}\left(\mathbf{N}_{2} \mathbf{G}_{1} \mathbf{N}_{1}\right)=\operatorname{Tr}\left(\mathbf{G}_{1} \mathbf{N}_{1} \mathbf{N}_{2}\right)=\operatorname{Tr}\left(\mathbf{G}_{1} \mathbf{M}_{1}\right) \\
& \mathbf{G}_{1} \mathbf{M}_{1}=\mathbf{G}_{1} \cos \phi+\mathbf{G}_{1} \mathbf{J}_{1} \sin \phi, \quad \phi=2 \pi \nu_{0}
\end{aligned}
$$

and therefore

$$
\cos 2 \pi \nu=\cos \phi+\frac{1}{2} G_{1} \beta_{1} \sin \phi
$$

and

$$
W=2(1-\cos 2 \pi \nu)=2(1-\cos \phi)-G_{1} \beta_{1} \sin \phi
$$


Now, carrying out the matrix multiplication in (38) we obtain the components of $\mathbf{D}-\mathbf{D}_{0}$. Thus

$$
D-D_{0}=\frac{G_{1} D_{1}}{W}\left\{B_{2}(1-\cos \phi)+\left(\beta_{1} A_{2}-\alpha_{1} B_{2}\right) \sin \phi\right\}
$$

and

$$
D^{\prime}-D_{0}^{\prime}=\frac{G_{1} D_{1}}{W}\left\{F_{2}(1-\cos \phi)+\left(\beta_{1} E_{2}-\alpha_{1} F_{2}\right) \sin \phi\right\}
$$

where $A_{2}, B_{2}, E_{2}, F_{2}$ are given by (10-13) and $\phi=2 \pi \nu_{0}$. Collecting terms we find

$$
\begin{gathered}
\beta_{1} A_{2}-\alpha_{1} B_{2}=\sqrt{\beta_{1} \beta_{2}} \cos \psi \\
\beta_{1} E_{2}-\alpha_{1} F_{2}=-\sqrt{\beta_{1} / \beta_{2}}\left(\alpha_{2} \cos \psi+\sin \psi\right)
\end{gathered}
$$

where

$$
\psi=\psi_{2}-\psi_{1}, \quad \psi_{2}=2 \pi \nu_{0}=\phi, \quad \beta_{2}=\beta_{0}, \quad \alpha_{2}=\alpha_{0}
$$

and equations (44-45) become

$$
\begin{gathered}
D-D_{0}=\frac{G_{1} D_{1}}{W} \sqrt{\beta_{0} \beta_{1}}\left\{\sin \psi+\sin \psi_{1}\right\} \\
D^{\prime}-D_{0}^{\prime}=\frac{G_{1} D_{1}}{W} \sqrt{\beta_{1} / \beta_{0}}\left\{\cos \psi-\cos \psi_{1}-\alpha_{0}\left(\sin \psi+\sin \psi_{1}\right)\right\}
\end{gathered}
$$

\section{Four-Quadrupole Perturbation}

Independent manipulation of the periodic dispersion and its derivative requires at least two perturbing quadrupoles and these will in general alter the machine tunes and other lattice parameters. In this section we show that by adding two more quadrupoles to a minimal scheme of two, we can manipulate the periodic dispersion vector at $s_{0}$ without affecting the horizontal lattice parameters there. The scheme is based on the pairing of quadrupoles employed in the gamma-transition-jump schemes of the AGS and other machines [3-6].

We consider four thin quadrupoles placed at points $s_{1}, s_{2}, s_{3}$, and $s_{4}$ such that

$$
s_{0}<s_{1}<s_{2}<s_{3}<s_{4}<s_{5}, \quad s_{5}=s_{0}+C
$$

where $C$ is the ring circumference. We then have

$$
\mathbf{T}_{0}=\mathbf{U}_{5} \mathbf{U}_{4} \mathbf{U}_{3} \mathbf{U}_{2} \mathbf{U}_{1}, \quad \mathbf{T}_{2}=\mathbf{U}_{2} \mathbf{U}_{1} \mathbf{U}_{5} \mathbf{U}_{4} \mathbf{U}_{3},
$$




$$
\begin{gathered}
\mathbf{T}_{4}=\mathbf{U}_{4} \mathbf{U}_{3} \mathbf{U}_{2} \mathbf{U}_{1} \mathbf{U}_{5}, \quad \mathbf{T}=\mathbf{U}_{5} \mathbf{R}_{4} \mathbf{U}_{4} \mathbf{R}_{3} \mathbf{U}_{3} \mathbf{R}_{2} \mathbf{U}_{2} \mathbf{R}_{1} \mathbf{U}_{1} \\
\mathbf{M}_{0}=\mathbf{N}_{5} \mathbf{N}_{4} \mathbf{N}_{3} \mathbf{N}_{2} \mathbf{N}_{1}, \quad \mathbf{M}_{2}=\mathbf{N}_{2} \mathbf{N}_{1} \mathbf{N}_{5} \mathbf{N}_{4} \mathbf{N}_{3} \\
\mathbf{M}_{4}=\mathbf{N}_{4} \mathbf{N}_{3} \mathbf{N}_{2} \mathbf{N}_{1} \mathbf{N}_{5}, \quad \mathbf{M}=\mathbf{N}_{5} \mathbf{Q}_{4} \mathbf{N}_{4} \mathbf{Q}_{3} \mathbf{N}_{3} \mathbf{Q}_{2} \mathbf{N}_{2} \mathbf{Q}_{1} \mathbf{N}_{1}, \\
\mathbf{d}_{0}=\mathbf{N}_{5} \mathbf{N}_{4} \mathbf{N}_{3} \mathbf{N}_{2} \mathbf{n}_{1}+\mathbf{N}_{5} \mathbf{N}_{4} \mathbf{N}_{3} \mathbf{n}_{2}+\mathbf{N}_{5} \mathbf{N}_{4} \mathbf{n}_{3}+\mathbf{N}_{5} \mathbf{n}_{4}+\mathbf{n}_{5}
\end{gathered}
$$

and

$$
\begin{aligned}
\mathbf{d} & =\mathbf{N}_{5} \mathbf{Q}_{4} \mathbf{N}_{4} \mathbf{Q}_{3} \mathbf{N}_{3} \mathbf{Q}_{2} \mathbf{N}_{2} \mathbf{Q}_{1} \mathbf{n}_{1}+\mathbf{N}_{5} \mathbf{Q}_{4} \mathbf{N}_{4} \mathbf{Q}_{3} \mathbf{N}_{3} \mathbf{Q}_{2} \mathbf{n}_{2}+ \\
& +\mathbf{N}_{5} \mathbf{Q}_{4} \mathbf{N}_{4} \mathbf{Q}_{3} \mathbf{n}_{3}+\mathbf{N}_{5} \mathbf{Q}_{4} \mathbf{n}_{4}+\mathbf{n}_{5}
\end{aligned}
$$

Now suppose the quadrupoles are arranged in pairs such that

$$
\sin \left(\psi_{2}-\psi_{1}\right)=0, \quad \sin \left(\psi_{4}-\psi_{3}\right)=0
$$

and

$$
\beta_{2} G_{2}+\beta_{1} G_{1}=0, \quad \beta_{4} G_{4}+\beta_{3} G_{3}=0 .
$$

It then follows from $(10-13)$ that

$$
B_{2}=B_{4}=0, \quad A_{2} G_{2}+F_{2} G_{1}=0, \quad A_{4} G_{4}+F_{4} G_{3}=0
$$

and therefore

$$
\mathbf{Q}_{4} \mathbf{N}_{4} \mathbf{Q}_{3}=\mathbf{N}_{4}, \quad \mathbf{Q}_{2} \mathbf{N}_{2} \mathbf{Q}_{1}=\mathbf{N}_{2} .
$$

The second of equations (55) then becomes

$$
\mathbf{M}=\mathbf{N}_{5} \mathbf{N}_{4} \mathbf{N}_{3} \mathbf{N}_{2} \mathbf{N}_{1}=\mathbf{M}_{0}
$$

and it follows that the horizontal tune and the horizontal lattice parameters at $s_{0}$ are left unchanged by the four quadrupoles. Using (61) in (57) we also have

$$
\mathbf{d}=\mathbf{N}_{5} \mathbf{N}_{4} \mathbf{N}_{3} \mathbf{N}_{2} \mathbf{n}_{1}+\mathbf{N}_{5} \mathbf{N}_{4} \mathbf{N}_{3} \mathbf{Q}_{2} \mathbf{n}_{2}+\mathbf{N}_{5} \mathbf{N}_{4} \mathbf{n}_{3}+\mathbf{N}_{5} \mathbf{Q}_{4} \mathbf{n}_{4}+\mathbf{n}_{5}
$$

and subtracting (56) from (63) we have

$$
\mathbf{d}-\mathbf{d}_{0}=\mathbf{N}_{5} \mathbf{N}_{4} \mathbf{N}_{3} \mathbf{G}_{2} \mathbf{n}_{2}+\mathbf{N}_{5} \mathbf{G}_{4} \mathbf{n}_{4} .
$$

Then since $\mathbf{M}=\mathbf{M}_{0}$ it follows from (7) and (32) that

$$
\mathbf{D}-\mathbf{D}_{0}=\left(\mathbf{I}-\mathbf{M}_{0}\right)^{-1}\left(\mathbf{d}-\mathbf{d}_{0}\right)=\frac{1}{W_{0}}\left(\mathbf{I}-\overline{\mathbf{M}}_{0}\right)\left(\mathbf{d}-\mathbf{d}_{0}\right)
$$


where

$$
W_{0}=2-\operatorname{Tr} M_{0}=2\left(1-\cos 2 \pi \nu_{0}\right)
$$

Thus

$$
\mathbf{D}-\mathbf{D}_{0}=\frac{1}{W_{0}}\left(\mathbf{I}-\overline{\mathbf{M}}_{0}\right)\left\{\mathbf{N}_{5} \mathbf{N}_{4} \mathbf{N}_{3} \mathbf{G}_{2} \mathbf{n}_{2}+\mathbf{N}_{5} \mathbf{G}_{4} \mathbf{n}_{4}\right\}
$$

and since

$$
\overline{\mathbf{M}}_{0} \mathbf{N}_{5} \mathbf{N}_{4} \mathbf{N}_{3}=\mathbf{N}_{5} \mathbf{N}_{4} \mathbf{N}_{3} \overline{\mathbf{M}}_{2}, \quad \overline{\mathbf{M}}_{0} \mathbf{N}_{5}=\mathbf{N}_{5} \overline{\mathbf{M}}_{4}
$$

we have

$$
\mathbf{D}-\mathbf{D}_{0}=\frac{1}{W_{0}} \mathbf{N}_{5} \mathbf{N}_{4} \mathbf{N}_{3}\left(\mathbf{I}-\overline{\mathbf{M}}_{2}\right) \mathbf{G}_{2} \mathbf{n}_{2}+\frac{1}{W_{0}} \mathbf{N}_{5}\left(\mathbf{I}-\overline{\mathbf{M}}_{4}\right) \mathbf{G}_{4} \mathbf{n}_{4}
$$

where

$$
\mathbf{n}_{2}=\mathbf{D}_{2}-\mathbf{N}_{2} \mathbf{D}_{1}, \quad \mathbf{n}_{4}=\mathbf{D}_{4}-\mathbf{N}_{4} \mathbf{D}_{3} .
$$

Carrying out the matrix multiplication in (69-70) we obtain the components of $\mathbf{D}-\mathbf{D}_{0}$. Collecting terms as for the case of a single quadrupole perturbation we find

$$
\begin{aligned}
D-D_{0}=\frac{G_{2} n_{2}}{W_{0}} \sqrt{\beta_{0} \beta_{2}}\left\{\sin \phi_{2}+\sin \psi_{2}\right\}+\frac{G_{4} n_{4}}{W_{0}} \sqrt{\beta_{0} \beta_{4}}\left\{\sin \phi_{4}+\sin \psi_{4}\right\} \\
D^{\prime}-D_{0}^{\prime}=\frac{G_{2} n_{2}}{W_{0}} \sqrt{\beta_{2} / \beta_{0}}\left\{\cos \phi_{2}-\cos \psi_{2}-\alpha_{0}\left(\sin \phi_{2}+\sin \psi_{2}\right)\right\} \\
+\frac{G_{4} n_{4}}{W_{0}} \sqrt{\beta_{4} / \beta_{0}}\left\{\cos \phi_{4}-\cos \psi_{4}-\alpha_{0}\left(\sin \phi_{4}+\sin \psi_{4}\right)\right\}
\end{aligned}
$$

where

$$
\phi_{2}=2 \pi \nu_{0}-\psi_{2}, \quad \phi_{4}=2 \pi \nu_{0}-\psi_{4}
$$

and

$$
n_{2}=D_{2}-A_{2} D_{1}-B_{2} D_{1}^{\prime}, \quad n_{4}=D_{4}-A_{4} D_{3}-B_{4} D_{3}^{\prime} .
$$

\section{Application to Booster}

Let us now apply the formulae of the previous sections to the manipulation of dispersion in the F6 straight section. We take $s_{0}$ to be the center of the F6 quadrupole and use the formulae to calculate $\mathbf{D}-\mathbf{D}_{0}$ there. For the 
case of a single quadrupole perturbation we take $s_{1}$ to be the center of the F2 quadrupole and imagine that a thin quadrupole with strength $G_{1}$ is placed there. We shall assume that alpha is zero at the centers of the F2 and F6 quadrupoles and that the horizontal beta function at both points has the value $B$. We assume further that the derivative of the periodic dispersion is zero at both points. Thus we have

$$
\alpha_{2}=\alpha_{1}=\alpha_{0}=0, \quad \beta_{2}=\beta_{1}=\beta_{0}=B
$$

and

$$
D_{2}^{\prime}=D_{1}^{\prime}=D_{0}^{\prime}=0 .
$$

Equations (49-50) then become

$$
\begin{aligned}
& D-D_{0}=\frac{B G_{1} D_{1}}{W}\left(\sin \psi+\sin \psi_{1}\right), \\
& D^{\prime}-D_{0}^{\prime}=\frac{G_{1} D_{1}}{W}\left(\cos \psi-\cos \psi_{1}\right),
\end{aligned}
$$

where

$$
W=2(1-\cos \phi)-B G_{1} \sin \phi, \quad \psi=\psi_{2}-\psi_{1}, \quad \psi_{2}=2 \pi \nu_{0}=\phi .
$$

These equations give the change in the periodic dispersion at F6 due to the thin quad at F2. Using (79) in (77), and solving for $B G_{1}$ we obtain

$$
B G_{1}=\frac{2\left(D-D_{0}\right)(1-\cos \phi)}{D_{1}\left(\sin \psi+\sin \psi_{1}\right)+\left(D-D_{0}\right) \sin \phi}
$$

which gives the quadrupole strength required to change the dispersion at F6 by $D-D_{0}$. If we want the perturbed dispersion $D$ to be zero, (80) becomes

$$
B G_{1}=\frac{2 D_{0}(\cos \phi-1)}{D_{1}\left(\sin \psi+\sin \psi_{1}\right)-D_{0} \sin \phi} .
$$

Now, if we take the unperturbed horizontal tune to be $\nu_{0}=4.8=24 / 5$, then the betatron phase advance per superperiod is $2 \pi \nu_{0} / 6=8 \pi / 5$ and the betatron phase advance between adjacent quadrupoles is approximately $\pi / 5$. The betatron phase advance between the centers of the F2 and F6 quadrupoles is then approximately $4 \pi / 5$. Thus we have

$$
\phi=48 \pi / 5, \quad \psi=4 \pi / 5, \quad \psi_{1}=44 \pi / 5
$$


and $(81)$ becomes

$$
B G_{1}=\frac{(-1.3820) D_{0}}{(1.1756) D_{1}+(.9511) D_{0}}
$$

Then taking $D_{0}=2.9$ meters and $D_{1}=1.4$ meters we have $G_{1} B=-0.91$, and taking $B=13.6$ meters we find that the required focal length for the thin quad at F2 is $G_{1}^{-1}=-14.9$ meters. This is to be compared with the focal length of approximately -3.6 meters for the horizontally focusing lattice quadrupoles. Thus, in order to make the periodic dispersion at F6 equal to zero, the strength of the F2 quadrupole must be increased by about 24 per cent. This is in agreement with the result obtained with the MAD code.

Let us now consider the four-quadrupole perturbation. We shall assume, as with the single quadrupole case, that

$$
\begin{gathered}
\alpha_{0}=\alpha_{1}=\alpha_{2}=\alpha_{3}=\alpha_{4}=\alpha_{5}=0, \\
\beta_{0}=\beta_{1}=\beta_{2}=\beta_{3}=\beta_{4}=\beta_{5}=B, \\
D_{0}^{\prime}=D_{1}^{\prime}=D_{2}^{\prime}=D_{3}^{\prime}=D_{4}^{\prime}=D_{5}^{\prime}=0 .
\end{gathered}
$$

Equations (71-74) then become

$$
\begin{gathered}
D-D_{0}=\frac{B G_{2} n_{2}}{W_{0}}\left\{\sin \phi_{2}+\sin \psi_{2}\right\}+\frac{B G_{4} n_{4}}{W_{0}}\left\{\sin \phi_{4}+\sin \psi_{4}\right\} \\
D^{\prime}-D_{0}^{\prime}=\frac{G_{2} n_{2}}{W_{0}}\left\{\cos \phi_{2}-\cos \psi_{2}\right\}+\frac{G_{4} n_{4}}{W_{0}}\left\{\cos \phi_{4}-\cos \psi_{4}\right\}
\end{gathered}
$$

where

$$
\phi_{2}=2 \pi \nu_{0}-\psi_{2}, \quad \phi_{4}=2 \pi \nu_{0}-\psi_{4}
$$

and

$$
n_{2}=D_{2}-D_{1} \cos \left(\psi_{2}-\psi_{1}\right), \quad n_{4}=D_{4}-D_{3} \cos \left(\psi_{4}-\psi_{3}\right)
$$

Taking $D=D^{\prime}=0$ and solving (87-88) for $G_{2} n_{2}$ and $G_{4} n_{4}$ we find

$$
G_{2} n_{2}=\frac{D_{0}\left(\cos \psi_{4}-\cos \phi_{4}\right)}{B \sin \left(\psi_{4}-\psi_{2}\right)}, \quad G_{4} n_{4}=\frac{-D_{0}\left(\cos \psi_{2}-\cos \phi_{2}\right)}{B \sin \left(\psi_{4}-\psi_{2}\right)}
$$

which give the quadrupole strengths required to make the periodic dispersion and its derivative equal to zero at the center of the F6 quadrupole. Now, as before, we take the unperturbed horizontal tune to be 
$\nu_{0}=4.8=24 / 5$, so that the betatron phase advance between adjacent quadrupoles is approximately $\pi / 5$. Then in order to satisfy equations (58) and (85) we must take $\psi_{4}-\psi_{3}$ and $\psi_{2}-\psi_{1}$ to be even multiples of $\pi$. (If they are odd multiples, then one quadrupole in each pair will be at a horizontal beta maximum and the other will be at a horizontal beta minimum.) As one possible arrangement we take $s_{1}, s_{2}, s_{3}, s_{4}$ to be the centers of quadrupoles $\mathrm{C} 6, \mathrm{D} 8, \mathrm{E} 2, \mathrm{~F} 4$ respectively. We then have

$$
\begin{gathered}
\psi_{1}=24 \pi / 5, \quad \psi_{2}=34 \pi / 5, \quad \psi_{3}=36 \pi / 5, \quad \psi_{4}=46 \pi / 5 \\
\phi_{2}=14 \pi / 5, \quad \phi_{4}=2 \pi / 5, \quad \psi_{4}-\psi_{2}=12 \pi / 5
\end{gathered}
$$

and equations (91) become

$$
G_{2}\left(D_{2}-D_{1}\right)=(-1.176) D_{0} / B, \quad G_{4}\left(D_{4}-D_{3}\right)=0 .
$$

(Note that $G_{4}\left(D_{4}-D_{3}\right)$ happens to be zero in this case because $\cos \phi_{2}=\cos \psi_{2}$.) Taking $D_{0}=D_{1}=D_{4}=2.9$ meters, $D_{2}=D_{3}=1.4$ meters, and $B=13.6$ meters we then have $G_{2}^{-1}=6.0$ meters, and according to (59) we must also have $G_{1}^{-1}=-6.0$ meters. Thus, increasing the strength of the $\mathrm{C} 6$ quadrupole by 60 per cent and decreasing that of the D8 quadrupole by the same amount will make the periodic dispersion and its derivative equal to zero in the F6 quadrupole.

\section{References}

1. J. Niederer, "BTA Lattice Matching", AGS/AD/Tech. Note No. 431, March 18, 1996.

2. J. Niederer, "More BTA Lattice Matching", AGS/AD/Tech. Note No. 440, August 12, 1996.

3. L. Ahrens, et. al., "A $\gamma_{t}$-Jump Scheme for the Brookhaven AGS", Accelerator Div. Tech. Note No. 265, September 26, 1986.

4. M.J. Syphers, et. al., "The AGS $\gamma_{t}$-Jump System", AGS/AD/94-5

Informal Report No. 60824, September 12, 1994.

5. W. Hardt, "Gamma-Transition-Jump Scheme of the CPS", CERN/MPS/DL 74-3, April 1974.

6. T. Risselada, "Gamma Transition Jump Schemes", CERN 91-04, May 8, 1991, pp. 161-174 